\title{
Article
}

\section{Robust estimators incorporating voltage and current phasors from PMUs}

\author{
Farhan Ammar Ahmad ${ }^{1,2, *}$ and Ibrahim Omar Habiballah ${ }^{1}$ \\ 1 Department of Electrical Engineering, King Fahd University of Petroleum and Minerals, Dhahran, Saudi Arabia.; \\ ibrahimh@kfupm.edu.sa(I.O.B) \\ 2 Department of Electrical Engineering, University of Management and Technology Lahore, Sialkot campus, Pakistan. \\ * Correspondence: fahran.sayal@post.umt.edu.pk; Tel.: +923234046006
}

Received: 20 January 2019; Accepted: 26 March 2019; Published: 4 April 2019.

\begin{abstract}
State Estimation is the backbone of modern electric power system and is used by almost all Energy Management Systems (EMS) in the world to ensure the real-time monitoring and secure operation of a power system. Phasor Measurement Unit (PMU) is most popular meter in today's electrical power industry because of its high refresh rates and measurement accuracy. Meanwhile, state estimation with only PMUs is not practical because of the very high initial installation cost. Consequently, the use of PMU meters along with conventional Supervisory Control and Data Acquisition (SCADA) meters can improve the performance of the state estimation. In this paper, phasor measurements (voltage and current phasors) are incorporated in two robust estimators: Weighted Least Absolute Value (WLAV) and Least Measurement Rejected (LMR). Further, we have investigated the importance of locating PMUs to save cost and improve the performance of state estimation. The performance of these two estimators after incorporating voltage and current phasors is investigated in terms of estimation accuracy of state variables and computational efficiency in the presence of different bad-data scenarios on IEEE-30 and IEEE-118 bus systems.
\end{abstract}

Keywords: Bad-data, least measurement rejected, phasor measurement units, robust estimators, state estimation, weighted least absolute value.

\section{Introduction}

$\mathbf{S}$ tate estimator is an algorithm that process raw and redundant conventional meter readings and other information i.e. network topology, circuit breaker status etc. to estimates the state of a power system [1-3]. State estimation is one of the basic tools used to ensure that the system is operational in secure mode and all constraints are satisfied. The installed systems at control center of electrical utilities process different measurements from different types of sensors and meters to estimate the overall operating condition of a power system. The measurements which are recorded wrongly because of the large noise, aging of a meter or calibration issue etc. are referred as bad-data [4] that affects the estimation process and results in the wrong estimation of the system's state variables (voltage magnitude and angle). Bad-data is mainly classified into two categories: (a) single bad-data and (b) multiple bad-data [5]. Multiple bad-data mostly occurs in very large systems and is strongly correlated to each other that poses a huge impact on the results of state estimation.

Weighted Least Square (WLS) is most popular state estimator deployed in electrical utilities and typically intakes reading from conventional meters including power flow meter, power injection meter, and voltage magnitude meter. The mathematical formulation of WLS is simple and has less computational burden, however, it is a non-robust state estimator because WLS fails to produce reliable estimation results even in the presence of single bad-data.

Weighted Least Absolute Value (WLAV) is a robust estimator, compared with WLS, however, it is susceptible to leverage points for certain configurations of meter distribution in a systems [6]. WLAV poses computational burden for large power systems that limits it utilization. For numerical stability and computational efficiency in linear programming (LP), the scaling technique is widely employed and proved an efficient tool [7] provided that scaling helps in reducing the effect of leverage points. In [8], a robust WLAV-T estimator is proposed to mitigate the effect of leverage points based on optimal transformation of 
associated rotation angles and scaling factor in systematic way, compared to heuristic approach. Further, the WLAV possesses auxiliary variables that reduce the convergence rate of the estimator, so Weighted Linear Least Square (WLLS) is proposed in [9] that possessed less number of variables than WLAV estimator and linear objective function.

Traditional state estimation is going through essential developments due to the innovation of Phasor Measurement Units (PMUs). State estimation problem is more easily formulated when there are adequate number of only PMUs installed in a power system because there is linear relationship between PMU's phasor measurements and state variables [10]. Least Absolute Value (LAV) estimator is computationally efficient and competitive with WLS if only phasor measurements are provided for state estimation and the strategic scaling method may be used to avoid biasness of leverage points [11,12]. In [10], the authors investigated the performance comparison between WLS and LAV when only PMUs were used in the presence of different bad-data scenarios and applied scaling technique to maintain the robustness of LAV. The accuracy, synchronization and redundancy of state estimation is improved by incorporating PMU phasor measurements with conventional SCADA measurement into the existing state estimator, however, there are many challenges in this implementation that are addressed with proposed solution [13]. In [14], the authors proposed a method to incorporate conventional SCADA having slow refresh rate of measurement and PMU phasor measurements with fast refresh rate in WLS estimator to keep track of the varying state of the power network. A hybrid state estimator [15] integrated with conventional Supervisory Control and Data Acquisition (SCADA) and PMU's phasor measurements is utilized for observability analysis and state estimation of a power system. In this hybrid estimator, there is switching between WLS and LAV estimator depending upon the availability of measurements from conventional SCADA or PMU's phasor measurements. The availability of measurements further depends upon the variation in refresh rate of SCADA and PMU's phasor measurements. The basic idea of hybrid state estimator is presented in [16]. A two stage linear estimator with only PMUs is proposed in [17] that is not only robust estimator but also computationally efficient because of even distribution of processing burden among different areas.

A PMU measures voltage phasor at a bus and current phasor of all incoming and outgoing flows at substation where it has been installed [18]. A modified non-linear WLS estimator incorporating voltage and current phasors in rectangular and polar coordinates is presented in [19] and both approaches have been compared and evaluated on IEEE-14 bus system with different bad-data scenarios. In [20], a two-stage state estimator combining both SCADA and PMU is proposed and authors have claimed higher estimation accuracy over only conventional SCADA meters. An approach to integrate the PMU technology into the existing SCADA systems to improve the accuracy of state variable is proposed, however, the proposed approach has been tested on very small power system without providing solid mathematical formulation [21]. Another technique to combine both SCADA and $\mu \mathrm{PMU}$ meters in a distribution system state estimation is presented, however, the authors have proposed this approach for WLS, which is a non-robust state estimator [22]. In [23], the authors have proposed an approach to integrate both SCADA and $\mu \mathrm{PMU}$ meters together, however, the proposed approach is applied for network topology analysis and tested on IEEE-33 bus system only. Furthermore, the authors have not provided computational efficiency of the proposed approach.

Least Measurement Rejected (LMR) is a robust estimator which associate a tolerance range with each provided measurement. LMR is solved using mixed integer programming approach and it rejects unreliable, corrupted or wrongly recorded measurements during estimation process and it is not susceptible to leverage points [24]. LMR is simple and effective state estimation approach. In [25], the authors have proposed an iterative tuning approach to choose the appropriate tolerance value of LMR for a certain measurement configuration. The authors of [26] have proposed a novel approach to tune and select the best value for tolerance parameter of LMR. In literature, the different authors have integrated PMUs into existing SCADA system in different estimation algorithms e.g. WLS, WLAV, however, PMU's phasor measurements have not been incorporated in LMR which is also the novelty of our work.

Though, the above authors were successful in implementing the integration of SCADA and PMUs, however, many of them have applied their proposed approaches on non-robust estimator or the chosen test case was small power system. Furthermore, many authors could not provide information about computational efficiency and number to iterations required to complete the estimation process. Our proposed technique has been applied on two robust state estimators: WLAV and LMR and evaluated on IEEE-30 and IEEE-118 bus systems in terms of state variables (voltage magnitude and angle), computational efficiency and number of 
iterations. Further, the objective of this paper is to improve the state estimation accuracy in the presence of different bad-data scenarios (single and multiple) by incorporating the voltage and current phasor from PMUs. The tolerance parameter of LMR estimator is chosen properly to reject large errors in conventional measurements. The final approach of achieving the objective is the proper selection of the PMUs locations to ensure best performance and accuracy.

The paper work is organized as follows. The section 2 covers the formulation and details about inclusion of voltage and current phasors in the state estimators and modified mathematical model of the state estimators. Performance validation of proposed technique is presented in section 3. Finally, section 4 concludes the paper with a brief summary.

\section{Inclusion of Voltage and Current Phasors into Robust State Estimators}

Once a PMU is installed at a bus in a power system, the voltage phasor of the bus and current phasor of all the branches connected to the bus is measured accurately. In this paper, these PMU's phasors are incorporated into the existing robust estimators and it was expected to achieve higher state estimation accuracy, compared with conventional measurements. When a phasor measurement is included in a state estimator, the weight of the phasor measurement must be increased because the PMU's measurement is highly accurate [10]. In WLAV estimator, there is a weight matrix where any measurement is assigned a specific weight corresponds to its accuracy, however, there is no weight matrix or covariance matrix in LMR estimator. The simulation analysis of our paper reveals that reducing the tolerance parameter of LMR associated with a measurement is equivalent to increasing the weight of the measurement in WLAV.

It is required to build a relationship between branch current flows in transmission lines and state variables to incorporate the current phasor in the state estimators. The proposed model will include all transmission lines and transformers between buses. An ampere flow in a branch is expressed as given below [27]:

$$
\begin{aligned}
I_{i j, \text { real }} & =\left(g_{i j}+g_{s i}\right) V m_{i} \cos \left(\theta_{i}\right)-g_{i j} V m_{j} \cos \left(\theta_{j}\right)-\left(b_{i j}+b_{s i}\right) V m_{i} \sin \left(\theta_{i}\right)+b_{i j} V m_{j} \sin \left(\theta_{j}\right) \\
I_{i j, \text { imag }} & =\left(g_{i j}+g_{s i}\right) V m_{i} \sin \left(\theta_{i}\right)-g_{i j} V m_{j} \sin \left(\theta_{j}\right)+\left(b_{i j}+b_{s i}\right) V m_{i} \cos \left(\theta_{i}\right)-b_{i j} V m_{j} \cos \left(\theta_{j}\right)
\end{aligned}
$$

where $I_{i j, \text { real }}$ and $I_{i j, i m a g}$ are rectangular components of the branch current flowing between bus $i$ and $j, V_{i}$, $V_{j}, \theta_{i}$ and $\theta_{j}$ are voltage magnitude and phase angle of bus $i$ and $j$ respectively, $g_{i j}$ and $b_{i j}$ are conductance and susceptance between bus $i$ and $j$ respectively, $g_{s i}$ and $b_{s i}$ are shunt conductance and susceptance respectively.

Practically, a PMU provides ampere measurements in polar coordinates rather than rectangular coordinates and can referred as direct measurements. However, it is better to use current phasors in rectangular coordinates, because the power flow measurements and power injection measurements are already in rectangular coordinates. In this regard, the ampere measurements should be converted to rectangular coordinates and utilized for state estimation. If the direct measurements are converted to rectangular coordinates, then error covariance values must be translated for rectangular coordinates. The relation between direct and indirect measurement is given by following equations:

$$
\begin{gathered}
I_{i j, i m a g}=I_{i j} \cos \left(\theta_{I_{i j}}\right) \\
I_{i j, i m a g}=I_{i j} \sin \left(\theta_{I_{i j}}\right)
\end{gathered}
$$

where $I_{i j}$ is branch current magnitude between bus $i$ and $j, \theta_{I_{i j}}$ is branch current phase angle between bus $i$ and $j$. The standard deviation of translated measurements can be calculated from below given Equations 5 and 6 [19], [28].

$$
\begin{gathered}
\sigma_{I_{i j, \text { real }}}=\sqrt{\left(\frac{\partial I_{i j, \text { real }}}{\partial I_{i j}}\right) \sigma_{I_{i j}}^{2}+\left(\frac{\partial I_{i j, \text { real }}}{\partial \theta_{i j}}\right) \sigma_{\theta_{I_{i j}}}^{2}} \\
\sigma_{I_{i j, \text { real }}}=\sqrt{\left(\cos \left(\theta_{I_{i j}}\right)\right)^{2} \sigma_{I_{i j}}^{2}+\left(I_{i j} \sin \left(\theta_{I_{i j}}\right)\right)^{2} \sigma_{\theta_{I_{i j}}}^{2}}
\end{gathered}
$$




$$
\begin{gathered}
\sigma_{I_{i j, i m a g}}=\sqrt{\left(\frac{\partial I_{i j, i m a g}}{\partial I_{i j}}\right) \sigma_{I_{i j}}^{2}+\left(\frac{\partial I_{i j, i m a g}}{\partial \theta_{i j}}\right) \sigma_{\theta_{i j}}^{2}} \\
\sigma_{I_{i j, i m a g}}=\sqrt{\left(\cos \left(\theta_{I_{i j}}\right)\right)^{2} \sigma_{I_{i j}}^{2}+\left(I_{i j} \sin \left(\theta_{I_{i j}}\right)\right)^{2} \sigma_{\theta_{I_{i j}}}^{2}}
\end{gathered}
$$

where $\sigma_{I_{i j, \text { real }}}$ and $\sigma_{I_{i j, i m a g}}$ are standard deviation of real and imaginary parts of current flows respectively, $\sigma_{I_{i j}}=0.02 p . u$ and $\sigma_{\theta_{I_{i j}}}=0.0017 \mathrm{rad}$ and are standard deviation of $I_{i j}$ and $\theta_{I_{i j}}$ respectively.

\subsection{Modified Weighted Least Absolute Value (WLAV) with Voltage and Current Phasors}

In this section, the mathematical formulation of modified WLAV incorporated with voltage and current phasors is presented. Usually, LP solving approach like simplex method or interior point method is used for WLAV. The performance of WLAV is very good for rejection of bad-data, however, it fails to provide reliable estimation results in the presence of leverage points [4]. There are numerous methods to identify leverage measurements [29] which is not the scope of this work.

A power system consists of $n$ buses with a specific network topology where $m$ meters are placed at different bus and branch locations to provide measurements from meters to state estimators through Remote Terminal Unit (RTU). The measurement vector $z$ of size $(m \times 1)$ is fed into a state estimator to obtain the state variable vector $x$ of size $n=(2 n \times 1)$. The non-linear function relating measurements to system state variables is given below:

$$
z=h(x)+e
$$

where $h(x)$ is the non-linear function relating measurements with the state vectors, and $e$ is the measurement error vector. Using the first order approximation for the Equation 7 , it can be written around some operating point $\hat{x}$ as

$$
\begin{gathered}
\triangle z=H(\hat{x}) \triangle x+e \\
H=\left[\begin{array}{c}
H_{i j} \\
H_{i n j} \\
H_{v} \\
H_{I_{i j}}
\end{array}\right] \\
H_{i j}=\left[\begin{array}{c}
\triangle P_{i j} \\
\triangle Q_{i j}
\end{array}\right]=\left[\begin{array}{ll}
\frac{\partial P_{i j}}{\partial \theta_{i}} & \frac{\partial P_{i j}}{\partial V m_{i}} \\
\frac{\partial Q_{i j}}{\partial \theta_{i}} & \frac{\partial Q_{i j}}{\partial V m_{i}}
\end{array}\right]\left[\begin{array}{c}
\triangle \theta_{i} \\
\triangle m_{i}
\end{array}\right] \\
H_{i n j}=\left[\begin{array}{cc}
\triangle P_{i n j} \\
\triangle Q_{i n j}
\end{array}\right]=\left[\begin{array}{ll}
\frac{\partial P_{i n j}}{\partial \theta_{i}} & \frac{\partial P_{i n j}}{\partial V m_{i}} \\
\frac{\partial Q_{i n j}}{\partial \theta_{i}} & \frac{\partial Q_{i n j}}{\partial V m_{i}}
\end{array}\right]\left[\begin{array}{c}
\triangle \theta_{i} \\
\triangle V m_{i}
\end{array}\right] \\
H_{v}=\left[\begin{array}{ll}
0 & \triangle \theta_{i} \\
1
\end{array}\right] \\
H_{I_{i j}=\left[V m_{i}\right.}=\left[\begin{array}{ll}
\triangle I_{i j, r e a l} \\
\triangle I_{i j, i m a g}
\end{array}\right]=\left[\begin{array}{ll}
\frac{\partial I_{i j, r e a l}}{\partial \theta_{i}} & \frac{\partial I_{i j, r e a l}}{\partial V m_{i}} \\
\frac{\partial I_{i j, i m a g}}{\partial \theta_{i}} & \frac{\partial I_{i j, i m a g}}{\partial V m_{i}}
\end{array}\right]\left[\begin{array}{c}
\triangle \theta_{i} \\
\triangle m_{i}
\end{array}\right]
\end{gathered}
$$

where $P_{i j}$ and $Q_{i j}$ are real and reactive power flow between bus $i$ and $j, P_{i n j}$ and $Q_{i n j}$ are real and reactive power injection between bus $i$ and $j$ respectively, $V m_{i}$ and $\theta_{i}$ are voltage magnitude and phase angle between at bus $i$, and $H$ is the modified Jacobian matrix as voltage and current phasors from PMUs are incorporated in it. 
The minimization objective function for WLAV is

$$
f(x)=\sum_{i=1}^{m} \frac{\left|\left(z_{i}-h_{i}(x)\right)\right|}{\sigma_{i}}
$$

where $\sigma_{i}$ is standard deviation of $i t h$ measurement. The standard deviation of PMU's measurements will be given different from the measurements of SCADA meters because PMU's measurements are considered more accurate, compared with measurements from SCADA. The values of standard deviation of SCADA and PMU's measurements are given in section 3. The minimization problem in 14 can be transformed into LP problem

$$
\min c^{T} \cdot Y
$$

Subject to

$$
\begin{gathered}
A \cdot Y=b \\
Y \geq 0
\end{gathered}
$$

where

$$
\begin{aligned}
& c^{T}=\left[0_{n} 0_{n} \frac{1}{\sigma_{p}} \frac{1}{\sigma_{p}}\right] \\
& 0_{n}=[0, \ldots, 0] \\
& \sigma_{p}=\left[\sigma_{1}, \sigma_{2}, \ldots, \sigma_{m}\right] \\
& Y^{T}=\left[\begin{array}{llll}
x_{u} & x_{v} & u & v
\end{array}\right] \\
& A=\left[H-H I_{m}-I_{m}\right] \\
& b=\Delta z
\end{aligned}
$$

where $x_{u}$ and $x_{v}$ are the components of state variables and both of these variables are size of $n=(2 N-1)$, $N$ is number of buses, $I_{m}$ is identity matrix of size $m x m$, and $m$ is number of measurements. The difference between $x_{u}$ and $x_{v}$ will provide values of state variables at each iteration.

\subsection{Modified Least Measurement Rejected with Voltage and Current Phasors}

A robust state estimation technique was proposed using mixed integer programming in [24], which correlates a specific tolerance value to each measurement in a power system. For each measurement, a tolerance value is defined which helps the estimator to reject unreliable or corrupted measurements during the estimation process. This robust estimation approach is invulnerable to leverage points even in pathological cases. The upper and lower tolerance values can be asymmetrical or symmetrical e.g. a power injection meter of $10 \mathrm{MW}$ may have upper tolerance value of $0.5 \mathrm{MW}$ and lower tolerance value of $0.75 \mathrm{MW}$, the measurement value taken from the power injection meter between (10.5MW and 9.25MW) will be considered as good and reliable. However, if the measurement value lies outside the specified range, then it will be suspected as corrupted or bad-data. This is an effective and trivial estimation approach and known as Least Measurement Rejected (LMR) and it is a variant of Least Median of Square (LMS). LMR minimizes the number of rejected measurements within a defined tolerance and its objective function is given below: 


$$
\begin{gathered}
K=\min \sum_{i=1}^{m} k_{i} \\
z_{i}-t_{i}-M k_{i} \leq h_{i}(x) \leq M k_{i}+t_{i}+z_{i}
\end{gathered}
$$

where $h_{i}(x)$ is measurement equation of $i t h$ meter, $t_{i}$ is tolerance value of $i t h$ measurement, $k_{i}$ is a binary number indicating whether the measurement error is within a specified tolerance limit or not, and $M$ is arbitrary large scalar value. The Equations 16 and 17 have been transformed into mixed integer programming problem and the formulation can be written as:

$$
\min c^{T} \cdot Y
$$

Subject to

$$
A . Y=b
$$

where

$$
\begin{gathered}
c^{T}=\left[\begin{array}{ll}
0_{n} & 1_{m}
\end{array}\right] \\
A=\left[\begin{array}{ll}
h & -M \\
h & -M
\end{array}\right] \\
B^{T}=[b+t b-t] \\
b=\triangle z \\
Y^{T}=\left[\begin{array}{ll}
\triangle x & k
\end{array}\right]
\end{gathered}
$$

where, $n$ is number of state variables, $H$ is modified Jacobean matrix explained in Equation $9, M$ is a vector of arbitrary large scaler value, $t$ is a vector that contains tolerance values of all provided measurements, $b$ is a vector of difference between estimated and loaded measurement values and $\triangle x$ is a vector of change is state variables.

\section{Performance Validation}

The method of voltage and current phasors inclusion into the existing robust state estimators is discussed in section 2 and is evaluated in the presence of different bad-data scenarios. Five different types of bad-data scenarios are considered in this paper, such as: (a) single bad-data (SBD) as power flow meter, (b) single bad-data (SBD) as a power injection meter, (c) single bad-data (SBD) as a voltage magnitude meter, (d) multiple non-interacting bad-data (MNIBD), and (e) multiple interacting bad-data (MIBD) as a combination of power flow meter, power injection meter, and voltage magnitude meter. This paper also investigates the importance of locating PMUs in a power system, however, optimal placement of PMUs is not the scope of this paper. Only two PMUs are placed heuristically in the considered test cases. The locations for bad-data scenarios is carefully chosen to ensure that the meter location selected for bad-data is not a critical location. The performance of robust state estimators is compared in terms of absolute error between actual and estimated voltage magnitudes and actual and estimated voltage angles, and computational efficiency. The actual values of voltage magnitude and angle are taken from load flow solution. The units for absolute voltage magnitude and angle are per unit and degrees respectively. The lower the absolute error, the better is the performance of the estimator. The proposed approach has been applied on IEEE-30 and IEEE-118 bus systems. The test cases are prepared on observable heuristic approach and each test case possesses different set of measurement types and different redundancy level. MATPOWER package is deployed to complete the execution of state estimation algorithm in MATLAB. WLAV and LMR have been solved using lp_solve package and mixed 
integer programming approach in MATLAB R2015a respectively. The simulation is performed on Intel Core i5, 4th generator processor with 4GB RAM. For LMR, $M=50000$ has been used for the IEEE-30 and IEEE-118 bus systems. The details of measurement types before and after the inclusion of PMUs are presented in Table 1 and Table 2 respectively.

Table 1. Measurement types

\begin{tabular}{||cc||}
\hline Measurement type & Description \\
\hline \hline$P F_{i-j}$ & Real power flow from bus $i$ to $j$ \\
$P F_{j-i}$ & Real power flow from bus $j$ to $i$ \\
$P G_{i n j}$ & Real power injection at bus $i$ \\
$Q F_{i-j}$ & Reactive power flow from bus $i$ to $j$ \\
$Q F_{j-i}$ & Reactive power flow from bus $j$ to $i$ \\
$Q G_{i n j}$ & Reactive power injection at bus $i$ \\
$V m_{i}$ & Voltage magnitude at bus $i$ \\
\hline
\end{tabular}

Table 2. Measurement types from PMUs

\begin{tabular}{||cc||}
\hline Measurement Type & Description \\
\hline \hline$\theta_{i}$ & Voltage Angle \\
$I_{i-j, r e a l}$ & Real current flow from bus $\mathrm{i}$ to $\mathrm{j}$ \\
$I_{i-j, \text { imag }}$ & Imaginary current flow from bus $\mathrm{i}$ to $\mathrm{j}$ \\
$I_{j-i, \text { real }}$ & Real current flow from bus $\mathrm{j}$ to $\mathrm{i}$ \\
$I_{j-i, \text { imag }}$ & Imaginary current flow from bus $\mathrm{j}$ to $\mathrm{i}$ \\
\hline
\end{tabular}

The values of standard deviation for SCADA and PMU's measurements are provided in Table 3 and the standard deviations of current phasors are calculated using Equations 5 and 6.

Table 3. Measurements standard deviation

\begin{tabular}{||ccc||}
\hline Measurement type & \multicolumn{2}{c||}{ Standard deviation } \\
\hline \hline & Without PMU & With PMU \\
\hline \hline$P F_{i-j}, P F_{j-i}, P G_{i n j}$ & 0.02 & 0.0002 \\
$Q F_{i-j}, Q F_{j-i}, Q G_{i n j}$ & 0.04 & 0.0004 \\
$V m_{i}$ & 0.01 & 0.0001 \\
\hline
\end{tabular}

Usually, the values of standard deviation are used to calculate the weighting factor for WLAV. After the inclusion of PMU's measurements in WLAV, the weights of those specific meter readings have to be increased. The simulated measurements for PMU's phasors are considered as the actual load flow values obtained from the power flow solution. In LMR, since there is no weighting factor in its mathematical formulation, the PMU's measurements are taken as actual load flow values and the tolerance value of those specific measurements has to be set as zero. In this case, the LMR provides the excellent estimation results, compared with other state estimators.

\subsection{IEEE 30 Bus System}

The meter distribution details for the test case are listed in Table 4 . The reference bus is slack bus ' 1 ' having zero-degree phase angle. The test case considered in this paper has only 126 SCADA meters. There are total 14 voltage magnitude meters in the test case, so there is wide-ranging choice for PMUs placement. In all cases for PMUs placement, one PMU is placed fixed at bus ' 1 ' to act as the reference PMU. The second location for PMU placement is chosen heuristically and it is ensured while placing PMU that the system is completely observable. All the critical measurements and sets are identified in the test case before simulating a bad-data at different types of meters. 
Table 4. IEEE 30 Bus - Meter distribution

\begin{tabular}{||cc||}
\hline Measurement type & Number of measurements \\
\hline \hline Real Power Flows & 41 \\
Real Power Injection & 16 \\
Reactive Power Flows & 40 \\
Reactive Power Injection & 15 \\
Voltage Magnitude & 14 \\
\hline
\end{tabular}

Computational time to complete a process or execute an algorithm is one of the major constraints in today's computing resources. The computation efficiency of WLAV and LMR for IEEE-30 bus system is shown in Table 5 and it is clearly reflected from the results that LMR requires lower computational time and a less number of iterations than WLAV. It can be noticed that the maximum time required by LMR to complete state estimation is lower than minimum time required by WLAV.

Table 5. IEEE 30 Bus - Computation efficiency of the state estimators

\begin{tabular}{||clcc||}
\hline State estimator & \multicolumn{2}{l||}{ Convergence time $(\mathrm{sec})$} & Number of iterations \\
\hline \hline & Min & Max & \\
\hline \hline WLAV & 0.31 & 0.36 & 4 \\
LMR & 0.17 & 0.28 & 2 \\
\hline
\end{tabular}

The results for absolute voltage magnitude (AVM) error with different bad-data scenarios are shown in Table 6. In the first column, 'NO PMU' means that there are only SCADA meters available in the test case. As already explained, one PMU is kept fixed at slack bus and another is relocated at different buses to get estimation results and highlight the importance of PMU placement. PMU-3 means that one PMU is fixed at slack bus and another is placed at bus 3 .

It can be noticed from the results in Table 6 that AVM error of 'NO PMU' is higher than all PMU placement cases i.e. PMU-3, PMU-5, PMU-10 and PMU-21 for both WLAV and LMR. However, any optimal location cannot be suggested as the optimal placement of PMUs is not the scope of this work. In this paper, the objective was to incorporate PMUs in existing state estimators and to compare the performance of WLAV and LMR in terms of computational efficiency and accuracy.

It can be observed from the results that inclusion of PMUs has improved the estimation accuracy of both WLAV and LMR estimators in all PMU placements which is reflected by the lower value of AVM error in all PMU placement cases than AVM of 'No PMU' case and LMR has better state estimation accuracy than WLAV.

The results for absolute voltage angle (AVA) error with different bad-data scenarios are shown in Table 7 and the angles are measured in degrees. It can be noticed from that AVA error of all PMU placement cases is lower than 'NO PMU' case for both WLAV and LMR. It is clearly reflected that inclusion of PMUs in the existing state estimators have not only improve voltage magnitude but also improved voltage angle for both WLAV and LMR. However, LMR has higher estimation accuracy as compared with WLAV in all PMU placement cases.

Table 6. IEEE 30 Bus - Absolute voltage magnitude (AVM) error

\begin{tabular}{||ccccccccccccc||}
\hline $\begin{array}{c}\text { PMU } \\
\text { location }\end{array}$ & White Noise & \multicolumn{4}{c}{$\begin{array}{c}\text { SBD as } \\
\text { power flow } \\
\text { at } P F_{2-5}\end{array}$} & $\begin{array}{c}\text { SBD as power } \\
\text { injection } \\
\text { at } P G_{2}\end{array}$ & $\begin{array}{c}\text { SBD as voltage } \\
\text { magnitude } \\
\text { at } V m_{12}\end{array}$ & $\begin{array}{c}\text { MNIBD at } \\
P F_{2-4} \\
P G_{5}, V m_{12}\end{array}$ & $\begin{array}{c}\text { MIBD at } \\
Q F_{6-9}, \\
Q G_{24}, V m_{12}\end{array}$ \\
\hline \hline & WLAV & LMR & WLAV & LMR & WLAV & LMR & WLAV & LMR & WLAV & LMR & WLAV & LMR \\
\hline \hline NO PMU & 0.0498 & 0.0200 & 0.0499 & 0.0200 & 0.0490 & 0.0212 & 0.0841 & 0.0664 & 0.0856 & 0.0559 & 0.0731 & 0.0584 \\
PMU-3 & 0.0394 & 0.0100 & 0.0394 & 0.0098 & 0.0328 & 0.0093 & 0.0655 & 0.0187 & 0.0650 & 0.0160 & 0.0500 & 0.0126 \\
PMU-5 & 0.0397 & 0.0104 & 0.0397 & 0.0104 & 0.0397 & 0.0198 & 0.0627 & 0.0201 & 0.0661 & 0.0200 & 0.0398 & 0.0139 \\
PMU-10 & 0.0207 & 0.0108 & 0.0207 & 0.0112 & 0.0205 & 0.0121 & 0.0311 & 0.0215 & 0.0230 & 0.0212 & 0.0242 & 0.0207 \\
PMU-21 & 0.0217 & 0.0169 & 0.0217 & 0.0169 & 0.0231 & 0.0163 & 0.0260 & 0.0219 & 0.0255 & 0.0247 & 0.0325 & 0.0197 \\
\hline
\end{tabular}


Table 7. IEEE 30 Bus - Absolute voltage angle (AVA) error

\begin{tabular}{|c|c|c|c|c|c|c|c|c|c|c|c|c|}
\hline \multirow[t]{2}{*}{$\begin{array}{c}\text { PMU } \\
\text { location }\end{array}$} & \multicolumn{2}{|c|}{ White Noise } & \multicolumn{2}{|c|}{$\begin{array}{l}\text { SBD as } \\
\text { power flow } \\
\text { at } P F_{2-5}\end{array}$} & \multicolumn{2}{|c|}{$\begin{array}{l}\text { SBD as power } \\
\text { injection } \\
\text { at } P G_{2}\end{array}$} & \multicolumn{2}{|c|}{$\begin{array}{l}\text { SBD as voltage } \\
\text { magnitude } \\
\text { at } V m_{12}\end{array}$} & \multicolumn{2}{|c|}{$\begin{array}{c}\text { MNIBD at } \\
P F_{2-4} \\
P G_{5}, V m_{12}\end{array}$} & \multicolumn{2}{|c|}{$\begin{array}{c}\text { MIBD at } \\
Q F_{6-9} \\
Q G_{24}, V m_{12}\end{array}$} \\
\hline & WLAV & LMR & WLAV & LMR & WLAV & LMR & WLAV & LMR & WLAV & LMR & $\overline{\text { WLAV }}$ & $\overline{\mathrm{LMR}}$ \\
\hline NO PMU & 2.8236 & 0.9927 & 2.9621 & 0.9927 & 2.2643 & 1.6630 & 3.5216 & 1.9322 & 4.0834 & 3.5875 & 4.4249 & 2.4865 \\
\hline PMU-3 & 1.1620 & 0.5947 & 1.1620 & 0.6011 & 1.2285 & 0.5570 & 1.1042 & 0.5036 & 1.1199 & 0.4136 & 1.1445 & 0.6171 \\
\hline PMU-5 & 1.0387 & 0.4735 & 1.0387 & 0.4715 & 1.2676 & 1.0270 & 1.1646 & 0.3330 & 1.0109 & 0.3414 & 1.0387 & 0.9417 \\
\hline PMU-10 & 0.6354 & 0.3040 & 0.6354 & 0.3061 & 0.5994 & 0.4121 & 1.0486 & 0.4871 & 0.7861 & 0.5908 & 0.5848 & 0.5200 \\
\hline PMU-21 & 0.8452 & 0.2509 & 0.8452 & 0.2313 & 1.0931 & 0.9487 & 0.8626 & 0.3419 & 0.9876 & 0.4605 & 0.8827 & 0.4030 \\
\hline
\end{tabular}

\subsection{IEEE 118 Bus System}

The meter distribution details for the test case have been shown in Table 8 . The test case considered in this paper has only 441 SCADA meters with a global redundancy of 1.87 . There are total 61 voltage magnitude meters, so there is wide choice for PMU placement. The PMUs are installed on randomly chosen buses. In IEEE-118 bus system, the slack bus is 69 and voltage phase angle is 30 degrees. One PMU is kept fixed at bus reference bus 69 and second PMU is relocated heuristically at different locations. It also has been ensured that system is completely observable, and all the critical measurements and sets have been identified before simulating bad-data scenarios on different types of SCAD measurements.

Table 8. IEEE 118 Bus - Measurement distribution

\begin{tabular}{||cc||}
\hline Measurement type & Number of measurements \\
\hline \hline Real Power Flows & 134 \\
Real Power Injections & 55 \\
Reactive Power Flows & 134 \\
Reactive Power Injections & 56 \\
Voltage Magnitude & 61 \\
\hline
\end{tabular}

The computational efficiency and number of iterations to complete state estimation are shown in Table 9. It can be seen from the presented results that LMR has lower computational time as compared with WLAV. However, both state estimators have same number of iterations but the overall computational efficiency of LMR is higher than WLAV as it requires lower completion time in seconds.

Table 9. IEEE 118 Bus - Computation efficiency of the state estimators

\begin{tabular}{||clcc||}
\hline State estimator & \multicolumn{2}{l||}{ Convergence time $(\mathrm{sec})$} & No. of iterations \\
\hline \hline & Min & Max & \\
\hline \hline WLAV & 6.40 & 9.17 & 6 \\
LMR & 3.73 & 4.26 & 6 \\
\hline
\end{tabular}

The results of AVM error for IEEE 118 bus system are shown in Table 10. It can be noticed from these results that inclusion of PMUs has improved the state estimation accuracy of both WLAV and LMR. However, LMR provided higher state estimation accuracy as compared with WLAV in all PMU placement cases.

Table 11 contains the results of AVA error for IEEE 118 bus system and the angles are measured in degrees. These results clearly reflect that inclusion of PMUs in the test case of IEEE 118 bus system has reduced the error in state estimation. Both state estimators, WLAV and LMR, have achieved higher estimation accuracy if compared with 'No PMU' case, however, the LMR has much lower values of AVA errors than WLAV in all PMU placements. Any optimal location for PMU placement cannot be advised from these results because optimal placement of PMUs is not the scope of this work and optimal placement problem has its own objective function with defined constraints. IEEE 118 bus is a large power system compared with IEEE 30 bus system and our proposed approach has successfully achieve higher level of accuracy even in large power system. 
Table 10. IEEE 118 Bus - Absolute voltage magnitude (AVM) error

\begin{tabular}{||ccccccccccccc||}
\hline $\begin{array}{c}\text { PMU } \\
\text { location }\end{array}$ & White Noise & \multicolumn{4}{c}{$\begin{array}{c}\text { SBD as } \\
\text { power flow } \\
\text { at } P F_{23}-32\end{array}$} & $\begin{array}{c}\text { SBD as power } \\
\text { injection } \\
\text { at } P G_{66}\end{array}$ & $\begin{array}{c}\text { SBD as voltage } \\
\text { magnitude } \\
\text { at } V m_{36}\end{array}$ & $\begin{array}{c}\text { MNIBD at } \\
P F_{4-11} \\
P G_{16}, V m_{3}\end{array}$ & $\begin{array}{c}\text { MIBD at } \\
P F_{34-36} \\
P G_{33}, V m_{18}\end{array}$ \\
\hline \hline & WLAV & LMR & WLAV & LMR & WLAV & LMR & WLAV & LMR & WLAV & LMR & WLAV & LMR \\
\hline \hline NO PMU & 0.2792 & 0.1976 & 0.2602 & 0.1775 & 0.2700 & 0.1816 & 0.2791 & 0.1976 & 0.2841 & 0.2090 & 0.2883 & 0.1816 \\
PMU-5 & 0.2035 & 0.1622 & 0.1910 & 0.1189 & 0.1992 & 0.1689 & 0.2034 & 0.1621 & 0.2135 & 0.1702 & 0.2135 & 0.1157 \\
PMU-12 & 0.2199 & 0.1786 & 0.2111 & 0.1715 & 0.2154 & 0.1811 & 0.2198 & 0.1856 & 0.2198 & 0.1872 & 0.2299 & 0.1514 \\
PMU-23 & 0.1952 & 0.1770 & 0.1951 & 0.1709 & 0.1904 & 0.1802 & 0.1951 & 0.1770 & 0.2034 & 0.1794 & 0.2066 & 0.1703 \\
PMU-30 & 0.1695 & 0.1197 & 0.1701 & 0.0901 & 0.1629 & 0.1017 & 0.1695 & 0.1196 & 0.1765 & 0.1462 & 0.1801 & 0.1261 \\
PMU-37 & 0.1737 & 0.1244 & 0.1655 & 0.1122 & 0.1692 & 0.0911 & 0.1737 & 0.1315 & 0.1876 & 0.1801 & 0.1740 & 0.1242 \\
PMU-49 & 0.1969 & 0.1048 & 0.1617 & 0.1176 & 0.1950 & 0.0482 & 0.1968 & 0.1048 & 0.2026 & 0.1706 & 0.2061 & 0.0847 \\
PMU-56 & 0.2121 & 0.1797 & 0.1781 & 0.1558 & 0.2131 & 0.1692 & 0.2121 & 0.1797 & 0.2179 & 0.1894 & 0.2213 & 0.1407 \\
PMU-77 & 0.2180 & 0.1446 & 0.1830 & 0.1328 & 0.2120 & 0.1365 & 0.2180 & 0.1445 & 0.2237 & 0.1577 & 0.2275 & 0.1032 \\
PMU-85 & 0.2177 & 0.1225 & 0.1823 & 0.1206 & 0.2108 & 0.1156 & 0.2176 & 0.1225 & 0.2232 & 0.1822 & 0.2271 & 0.1408 \\
PMU-94 & 0.2083 & 0.1055 & 0.1729 & 0.0879 & 0.2014 & 0.1018 & 0.2082 & 0.0869 & 0.2138 & 0.1250 & 0.2177 & 0.0896 \\
PMU-105 & 0.2196 & 0.1115 & 0.1842 & 0.0988 & 0.2127 & 0.1043 & 0.2195 & 0.1150 & 0.2251 & 0.1829 & 0.2290 & 0.1264 \\
PMU-110 & 0.2188 & 0.1370 & 0.1834 & 0.1281 & 0.2119 & 0.0946 & 0.2188 & 0.1369 & 0.2243 & 0.1580 & 0.2282 & 0.0827 \\
\hline
\end{tabular}

Table 11. IEEE 118 Bus - Absolute voltage angle (AVA) error

\begin{tabular}{|c|c|c|c|c|c|c|c|c|c|c|c|c|}
\hline \multirow[t]{2}{*}{$\begin{array}{c}\text { PMU } \\
\text { location }\end{array}$} & \multicolumn{2}{|c|}{ White Noise } & \multicolumn{2}{|c|}{$\begin{array}{c}\text { SBD as } \\
\text { power flow } \\
\text { at } P F_{23-32} \\
\end{array}$} & \multicolumn{2}{|c|}{$\begin{array}{c}\text { SBD as power } \\
\text { injection } \\
\text { at } P G_{66}\end{array}$} & \multicolumn{2}{|c|}{$\begin{array}{l}\text { SBD as voltage } \\
\text { magnitude } \\
\text { at } V m_{36}\end{array}$} & \multicolumn{2}{|c|}{$\begin{array}{c}\text { MNIBD at } \\
P F_{4-11} \\
P G_{16}, V m_{3} \\
\end{array}$} & \multicolumn{2}{|c|}{$\begin{array}{c}\text { MIBD at } \\
P F_{34-36} \\
P G_{33}, V m_{18} \\
\end{array}$} \\
\hline & WLAV & LMR & $\overline{\text { WLAV }}$ & " LMR & $\overline{\text { WLAV }}$ & 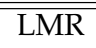 & $\overline{\text { WLAV }}$ & 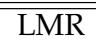 & $\overline{~ W L A V}$ & "LMR & WLAV & 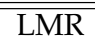 \\
\hline NO PMU & 6.5645 & 5.7658 & 8.4241 & 6.9760 & 6.9005 & 6.2859 & 6.5645 & 5.7658 & 8.7292 & 8.4180 & 8.8401 & 7.8542 \\
\hline PMU-5 & 4.8424 & 4.1135 & .5419 & 4.4315 & 4.1214 & 4.0842 & 4.8424 & 4.1136 & 7.1270 & 6.2995 & 6.6821 & 6.5527 \\
\hline PMU-12 & 5.6592 & 4.1274 & 4.9530 & 4.7770 & 5.0185 & 4.2916 & 5.6592 & 4.1614 & 7.6667 & 6.6985 & 7.7004 & 7.4777 \\
\hline PMU-23 & 5.1023 & 3.9799 & 5.1023 & 4.4299 & 4.4990 & 4.0794 & 5.1023 & 3.9799 & 7.4183 & 6.7431 & 8.1718 & 6.9019 \\
\hline PMU-37 & 4.9576 & 3.8861 & 6.7908 & 4.3050 & 4.5046 & 4.0361 & 4.9576 & 3.8754 & 6.4610 & 6.4533 & 4.2436 & 3.6609 \\
\hline PMU-49 & 4.9691 & 3.3357 & 4.8502 & 4.1246 & 4.6477 & 4.1873 & 4.9691 & 3.3368 & 7.8704 & 5.5164 & 6.6806 & 6.6307 \\
\hline PMU-56 & 4.7916 & 3.6311 & 6.8126 & 4.4770 & 5.0280 & 4.7945 & 4.7916 & 3.6311 & 7.6820 & 6.1747 & 7.4555 & 6.6350 \\
\hline PMU-77 & 5. & 4.1643 & 7.6845 & 4.3710 & 4.8917 & 4.8848 & 5.1490 & 4.1636 & 7.8193 & 6.8693 & 7.9457 & 7.1038 \\
\hline PMU-85 & 2203 & 3.6269 & 7.6517 & 4.6830 & 4.8087 & 4.5386 & 5.2203 & 3.6269 & 7.8273 & 6.3858 & 7.5832 & 7.1190 \\
\hline & 897 & 3.7719 & 7.9211 & 4.0410 & 5.0780 & 4.0052 & 5.4897 & 3.4912 & 8.0966 & 6.2631 & 7.3938 & 7.3884 \\
\hline PMU-105 & 5.0139 & 3.9057 & 7.4453 & 4.7130 & 4.6023 & 4.0723 & 5.0139 & 3.9050 & 7.6209 & 6.4835 & 7.5176 & 6.9126 \\
\hline PMU-110 & 4.9884 & 3.8172 & 7.4198 & 4.7730 & 4.5768 & 4.4168 & 4.9884 & 3.8172 & 7.5954 & 6.0187 & 7.6356 & 6.8871 \\
\hline
\end{tabular}

\section{Conclusion and Future Directions}

In this paper, the proposed approach to incorporate both voltage and current phasors from PMUs into the existing robust estimators: WLAV and LMR is successfully implemented and evaluated. The use of LMR estimator in the presence of voltage and current phasors coming from different locations is investigated provided significant improvement in the accuracy of the state estimation. The overall performance for LMR estimator compared with WLAV estimator proved to be better irrespective of the bad-data presence and test case size. Besides of the single bad-data cases, multiple interacting and non-interacting bad-data cases were also investigated that clearly reflected the robustness of LMR estimator and better estimation results compared with WLAV estimator. The working principle of LMR estimator depends upon proper tolerance level that assists in rejection of unreliable or corrupted measurement values as explained in the section 2. In this paper, the tolerance value of LMR estimator is tuned iteratively. The accurate meter readings from PMUs were easily incorporated into WLAV estimator by using covariance matrix. But for the LMR estimator, it was not possible to apply such higher weights to PMU meter readings like WLAV. The simulation study concluded into a pertinent contribution that the tolerance should be kept zero for all PMU meter readings. The accuracy of the estimator also depends upon the proper selection of the buses where the new PMUs are to be installed. This issue is carefully addressed in the paper for the test cases of IEEE 30 and IEEE 118 bus system. 


\section{Acknowledgements}

The authors acknowledge the support of King Fahd University of Petroleum and Minerals, Dhahran, Saudi Arabia.

Author Contributions: All authors contributed equally to the writing of this paper. All authors read and approved the final manuscript.

Conflicts of Interest: "The authors declare no conflict of interest."

\section{References}

[1] Schweppe, F. C., \& Wildes, J. (1970). Power system static-state estimation, Part I: Exact model. IEEE Transactions on Power Apparatus and systems, PAS-89(1), 120-125.

[2] Schweppe, F. C., \& Rom, D. B. (1970). Power system static-state estimation, Part II: Approximate model. IEEE Transactions on Power Apparatus and Systems, PAS-89(1), 125-130.

[3] Schweppe, F. C. (1970). Power system static-state estimation, Part III: Implementation. IEEE Transactions on Power Apparatus and systems, PAS-89(1), 130-135.

[4] Abur, A. (1990). A bad data identification method for linear programming state estimation. IEEE Transactions on Power Systems, 5(3), 894-901.

[5] Nian-De, X., Shi-Ying, W., \& Er-Keng, Y. (1982). A new approach for detection and identification of multiple bad data in power system state estimation. IEEE Transactions on Power Apparatus and Systems, PAS-101(2), 454-462.

[6] Xiaoli, Y., Zongshuai, H., Rusen, F., Haotian, X., Heng, Y., Yong, W., \& Xiuxia, T. (2015, April). Weighted least squares state estimation based on the optimal weight. In 2015 Third International Conference on Technological Advances in Electrical, Electronics and Computer Engineering (TAEECE) (pp. 12-16). IEEE.

[7] Celik, M. K., \& Abur, A. (1992). Use of scaling in WLAV estimation of power system states. IEEE Transactions on Power Systems, 7(2), 684-692.

[8] Celik, M. K., \& Abur, A. (1992). A robust WLAV state estimator using transformations. IEEE Transactions on Power Systems, 7(1), 106-113.

[9] Mahaei, S. M., \& Navayi, M. R. (2014). Power System State Estimation with Weighted Linear Least Square. International Journal of Electrical \& Computer Engineering (2088-8708), 4(2), 169-178.

[10] Gol, M., \& Abur, A. (2014). LAV based robust state estimation for systems measured by PMUs. IEEE Transactions on Smart Grid, 5(4), 1808-1814.

[11] Abur, A. (2015, July). Use of PMUs in WLS and LAV based state estimation. In 2015 IEEE Power \& Energy Society General Meeting (pp. 1-1). IEEE.

[12] Gol, M., \& Abur, A. (2012, September). PMU based robust state estimation using scaling. In 2012 North American Power Symposium (NAPS) (pp. 1-5). IEEE.

[13] Zhou, M., Centeno, V. A., Thorp, J. S., \& Phadke, A. G. (2006). An alternative for including phasor measurements in state estimators. IEEE transactions on power systems, 21(4), 1930-1937.

[14] Glavic, M., \& Van Cutsem, T. (2013). Reconstructing and tracking network state from a limited number of synchrophasor measurements. IEEE Transactions on Power Systems, 28(2), 1921-1929.

[15] Gol, M., \& Abur, A. (2015). A hybrid state estimator for systems with limited number of PMUs. IEEE Transactions on Power Systems, 30(3), 1511-1517.

[16] Gol, M., Abur, A., \& Galvan, F. (2013, October). Rapid tracking of bus voltages using synchro-phasor assisted state estimator. In IEEE PES ISGT Europe 2013 (pp. 1-5). IEEE.

[17] $\mathrm{Xu}, \mathrm{C} .$, \& Abur, A. (2018). A fast and robust linear state estimator for very large scale interconnected power grids. IEEE Transactions on Smart Grid, 9(5), 4975-4982.

[18] Bi, T. S., Qin, X. H., \& Yang, Q. X. (2008). A novel hybrid state estimator for including synchronized phasor measurements. Electric Power Systems Research, 78(8), 1343-1352.

[19] Korres, G. N., \& Manousakis, N. M. (2011). State estimation and bad data processing for systems including PMU and SCADA measurements. Electric Power Systems Research, 81(7), 1514-1524.

[20] Ortiz, G. A., Colome, D. G., \& Puma, J. J. Q. (2016, October). State estimation of power system based on SCADA and PMU measurements. In 2016 IEEE Andescon (pp. 1-4). IEEE.

[21] Bentarzi, H., Tsebia, M., \& Abdelmoumene, A. (2018, April). PMU based SCADA enhancement in smart power grid. In 2018 IEEE 12th International Conference on Compatibility, Power Electronics and Power Engineering (CPE-POWERENG 2018) (pp. 1-6). IEEE.

[22] Santos, R. Z. S., \& Orillaza, J. R. C. (2018, May). Distribution System State Estimator Using SCADA and $\mu$ PMU Measurements. In 2018 IEEE Innovative Smart Grid Technologies-Asia (ISGT Asia) (pp. 558-562). IEEE. 
[23] Zhang, X., Li, Y., Yang, C., Wang, S., Xie, W., \& Ling, P. (2018, November). Topology Analysis of Distribution Network Based on $\mu \mathrm{PMU}$ and SCADA. In 2018 International Conference on Power System Technology (Powercon) (pp. 3427-3433). IEEE.

[24] Irving, M. R. (2008). Robust state estimation using mixed integer programming. IEEE Transactions on Power Systems, 23(3), 1519-1520.

[25] Ahmad, F. A., Shahriar, M. S., Habiballah, I. O., \& Haider, A. (2018, February). State Estimation Accuracy of Tuned Least Measurement Rejected Estimator. In 2018 International Conference on Electrical Engineering (ICEE) (pp. 1-6). IEEE.

[26] Shahriar, M. S., Habiballah, I. O., \& Ahmad, F. A. (2018, September). Appropriate Tolerance Value Selection of Least Measurement Rejected Algorithm for Robust Power System State Estimation. In 2018 North American Power Symposium (NAPS) (pp. 1-6). IEEE.

[27] Bi, T. S., Qin, X. H., \& Yang, Q. X. (2008). A novel hybrid state estimator for including synchronized phasor measurements. Electric Power Systems Research, 78(8), 1343-1352.

[28] Rousseeuw, P. J., \& Leroy, A. M. (2005). Robust regression and outlier detection (Vol. 589). Hoboken, New Jersey: John wiley \& sons.

[29] Mili, L., Cheniae, M. G., Vichare, N. S., \& Rousseeuw, P. J. (1996). Robust state estimation based on projection statistics [of power systems]. IEEE Transactions on Power Systems, 11(2), 1118-1127. 